\title{
How To Maintain Maximal Straight Path Running Speed on a Curved Path in Sprint Events
}

\author{
by \\ Hayato Ohnuma ${ }^{1}$ Masanobu Tachi², Akihito Kumano ${ }^{3}$, Yuichi Hirano ${ }^{4}$
}

\begin{abstract}
This study aims to clarify the ideal technique for running on a curved path during sprinting events. Participants were twelve male track and field athletes including long jumpers and sprinters. The participants performed a 60-m sprint with maximal effort on straight and curved paths. Participants were divided into "good curve runners" and "poor curve runners" according to the curved path running speed relative to that of the straight path. Kinematic variables and ground reaction forces (GRFs) were registered and compared between the groups and paths. The running speed, step length, and flight distance of the outside leg on the curved path were lower than on the straight path only in poor curve runners. The medial-lateral GRF and impulse showed an increase during curved path running for both groups. However, the maximum posterior GRF and impulse decreased only in poor curve runners. The ideal technique for running on a curved path is to maintain the same kinematics and kinetics in the sagittal plane as on a straight path.
\end{abstract}

Key words: curved path, running technique, maximal running speed, sprinting.

\section{Introduction}

In sprinting events, maximal running speed largely affects sprint performance (Mackala, 2007). Previous studies have clarified some biomechanical factors that contribute to improving the running speed on a straight path (Brughelli et al., 2011; Hunter et al., 2004; Morin et al., 2012; Nummela et al., 2007; Weyand et al., 2000). In sprinting events, except for the 100-meter dash, 110-meter hurdles, and 100-meter hurdles, sprinters should run on both straight and curved paths at maximum or near maximum running speed. Therefore, to enhance sprint performance in most sprinting events, it is important to improve running speed on both straight and curved paths. However, it is generally accepted that the running speed on curved paths tends to be slower than on straight paths (Chang and Kram, 2007; Churchill et al., 2015a, 2015b; Greene, 1985; Stoner and Ben-Sira, 1979; Usherwood and Wilson, 2006).
Some previous studies attempted to clarify the reason for this detrimental effect of a curved path on maximal running speed. Stoner and Ben-Sira (1979) compared 20-m straight path and curved path running and indicated that on curved paths, the running speed was slower, and the spatiotemporal variables differed between legs. Usherwood and Wilson (2006) demonstrated that the main factor limiting the running speed on curved paths was an increase of the stance phase. The ground reaction force during curved and straight paths has been compared in previous studies (Chang and Kram, 2007; Churchill et al., 2015b; Hamill et al., 1987; Luo and Stefanyshyn, 2012; Smith et al., 2006). These studies showed that on a curved path, the ground reaction force differed between legs. The vertical ground reaction force tended to decrease in the leg that was inside during curved path running. However, the mediolateral component of the horizontal

1 - Department of Sports Science, Japan Institute of Sports Science, Tokyo, Japan.

2 - Faculty of Education, Nara University of Education, Nara, Japan.

3 - School of Physical Education, Tokai University, Hiratsuka, Japan .

4 - Faculty of Sports and Health Studies, Hosei University, Machida, Japan. 
ground reaction force of the inside leg was greater than of the outside leg.

These previous studies aimed to reveal the differences in sprint movement between straight and curved paths, and to clarify the factors that affected the running speed on curved paths. Most of the studies consistently showed that the running speed on a curved path was slower than on a straight path. On the other hand, Alt et al. (2015) indicated that there were no significant differences in running speed between straight and curved paths. Therefore, the extent of the decline in maximum running speed on a curved path in relation to a straight path differs among runners, i.e., there are good curve runners and poor curve runners. Running speed on a curved path is considered to be determined by maximum running speed or sprint ability on the straight path, and the cornering technique to change direction of movement along the curve without decreasing running speed. Therefore, knowledge of the differences in biomechanical characteristics between good and poor curve runners could lead to a better understanding of the technique for running faster on curved paths during sprinting events.

By comparing the differences in biomechanical characteristics between good and poor curve runners, this study aimed to clarify the ideal technique for running on a curved path during sprinting events.

\section{Methods}

\section{Participants}

Twelve male track and field athletes including long jumpers and sprinters (age $20.3 \pm$ 0.9 years, body height $1.74 \pm 0.04 \mathrm{~m}$, body mass $66.7 \pm 4.6 \mathrm{~kg}$ ) volunteered for this study. None of the participants reported any musculoskeletal injuries at the time of testing. Approval to undertake this study was given by the Nara University of Education Ethics Committee. Written informed consent was obtained from each participant.

\section{Measures}

The trials were $60 \mathrm{~m}$ sprint running on straight and curved paths. This experiment was conducted in the laboratory with all-weather pavement. Detrimental effects of curvature on running speed tend to be larger on a small radius (Greene, 1985; Quin, 2009). The radius of curvature was $37.9 \mathrm{~m}$, which corresponds to the most inside lane of a typical 400-m track. Reflective markers were placed on body landmarks based on Plug-in-Gait protocols (Davis et al., 1991; Kadaba et al., 1990). The measurement section was $45 \mathrm{~m}$ from the start on both paths. Three-dimensional positional data of the markers were recorded using a motion capture system operating at $250 \mathrm{~Hz}$ with 15 infrared cameras (Vicon-MX, Oxford Metrics Ltd., Oxford, UK). Two force-plate systems (Type 9286B, Kistler Inc., Winterthur, Switzerland) were used to sample ground reaction force (GRF) data at $1000 \mathrm{~Hz}$. The positional data and GRF data were synchronized using Vicon Nexus software (ver. 1.7.1, Oxford Metrics Ltd., Oxford, UK). In this study, we used only two force-plate systems. Therefore, the kinematic data and GRF data for each leg were obtained in separate trials. The participants performed 8 trials, two for each leg and path during the same days. Between trials, participants rested for more than ten minutes to offset the effect of fatigue on running speed. Global coordinate system directions were set as $\mathrm{X}$-axis for the medial-lateral direction, $Y$-axis for the anterior-posterior direction, and Z-axis for the vertical direction. On a curved path, the global coordinate system was translated to a local coordinate system in which the $X$-axis was defined as a radial-to-curved path, and the $\mathrm{Y}$-axis was a tangential-to-curved path.

\section{Procedures}

Vicon Nexus software was used to reconstruct the positions of each reflective marker in a three-dimensional graphical environment. Every marker on the participant was labeled according to the body landmark to which it was attached or according to the cluster to which it belonged, based on Plug-in Gait-protocols. The positional data and GRF data were smoothed using a fourth-order Butterworth digital filter with cutoff frequencies of 12 and $50 \mathrm{~Hz}$, respectively. The center of gravity was calculated using body segment variables, which were based on Jensen's mathematical modeling of Japanese athletes (Ae et al., 1992). The lower limb angle and angular velocity were calculated from these threedimensional coordinate values. Kinematic and GRF data of the fastest trial for each leg and each path were used for detailed analyses. All data processing was performed using MATLAB 
software (R2013a, The MathWorks Inc., Natick, MA, USA).

Spatiotemporal variables

Spatiotemporal variables were calculated for left and right steps on both paths. In accordance with previous studies (Churchill et al., 2015a, 2015b; Stoner and Ben-Sira, 1979), a right (outside) step was defined from right foot touchdown to left foot touchdown and vice versa for a left (inside) step. Running speed for each path was calculated by averaging the running speed for inside and outside steps. Participants were separated into good or poor curved path running groups based on the running speed on the curved path relative to the straight path.

Lower limb movements

Lower limb movements in the sagittal plane during the stance phase were calculated for left and right steps on both paths. Lower limb joint angles on foot touchdown and foot takeoff, minimum knee and ankle joint angles, and maximum lower limb joint flexion (dorsiflexion) and extension (plantarflexion) angular velocities were estimated.

Ground reaction force

The maximum and minimum GRF and the impulse for each coordinate were calculated for left and right steps. The impulse of the anterior-posterior component was divided into eccentric and concentric phases (Mero and Komi, 1986).

\section{Statistical analysis}

The Mann-Whitney U test was used to compare body characteristics and analyze variables between good and poor groups. In addition, the Wilcoxon signed-rank test was used to separately identify significant differences between straight and curved path running. For multiple comparisons, we used a Bonferroni correction to set the significance level at $p<0.05$, and Cohen's d was used to describe effect size (Cohen, 1992). All statistical analyses were performed using IBM SPSS Statistics software (v. 22.0, SPSS Inc., Chicago, Illinois, USA).

\section{Results}

The division of participants into good and poor curve runners

Figure 1 shows the running speed of all participants on straight and curved paths. The participants were equally divided into good and poor curved path running groups based on the percent difference of the running speed on both paths. In addition, percent difference of the running speed in the poor curved path running group was lower than in the good curved path running group $(p<0.01, d=5.82)$. There were no significant differences in body characteristics between groups (Good: body height $1.72 \pm 0.02 \mathrm{~m}$, body mass $64.9 \pm 2.5 \mathrm{~kg}$; Poor: body height $1.77 \pm$ $0.06 \mathrm{~m}$, body mass $67.6 \pm 5.9 \mathrm{~kg}$ ).

Spatiotemporal variables

Table 1 shows the spatiotemporal variables for each leg and path. The running speed on the curved path was slower than on the straight path in the poor curved path running group ( $p=0.03, d=1.24)$. The step frequency, stance time, and flight time did not differ between the groups or the paths. However, step length ( $p=$ $0.03, d=1.32)$ and flight distance $(p=0.03, d=1.42)$ of the outside leg on the curved path were significantly lower than on the straight path in poor curve runners. There were no significant differences in spatiotemporal variables between the groups.

Lower limb movements

Table 2 shows the lower limb movements for each leg during stance phases. There were no significant differences in lower limb movements during the flight phase between the paths and groups during the flight phase. During the stance phase, the hip joint angle at foot release for the outside leg ( $p=0.03, d=0.79)$ on the curved path was significantly smaller than on the straight path in poor curve runners. Furthermore, minimum knee $(p=0.03, d=0.93)$ and ankle joint angles $(p=$ $0.03, d=2.26$ ), and maximum knee joint extension angular velocity ( $p=0.03, d=0.30$ ) of the inside leg on the curved path were significantly smaller and faster than on the straight path in the poor curve runners. On the other hand, the hip joint angle at foot contact for both legs on the curved path was significantly smaller than on the straight path in both groups (Good Inside leg: $p=0.03, d=$ 0.79; Good Outside: $p=0.03, d=0.32$; Poor Inside: $p=0.03, d=0.79$; Poor Outside: $p=0.03, d=0.57$ ). There were no significant differences in lower limb movements during the stance phase between the two groups.

Ground reaction force

Table 3 shows the ground reaction forces for each leg. There were no significant differences in 
the vertical component between the groups or the paths. On the other hand, the maximum posterior GRF ( $p=0.03 ; d=0.92)$ and the impulse $(p=0.03, d$ $=2.14$ ) for the outside leg on the curved path were significantly lower than on the straight path in the poor curved runners group. Additionally, the maximum (Good-inside: $p=0.03$; $d=3.78$; Goodoutside: $p=0.03 ; d=2.32$; Poor-inside: $p=0.03 ; d=$ 2.56; Poor-outside: $p=0.03 ; d=1.78$ ) and minimum medial GRF (Good-inside: $p=0.03$; $d=$
2.61; Good-outside: $p=0.03$; $d=1.39$; Poor-inside: $p=0.03 ; d=2.65$; Poor-outside: $p=0.03 ; d=1.47$ ) and the impulse (Good-inside: $p=0.03$; $d=4.92$; Good-outside: $p=0.03$; $d=3.56$; Poor-inside: $p=$ 0.03; $d=2.96$; Poor-outside: $p=0.03 ; d=1.88$ ) for both legs on the curved path were significantly greater than on the straight path in both groups. There were no significant differences in GRF during the stance phase between the two groups.

Table 1

Differences in spatiotemporal variables

\begin{tabular}{lccccc}
\hline & & \multicolumn{2}{c}{ Good } & \multicolumn{2}{c}{ Poor } \\
\cline { 3 - 6 } & & straight & curved & straight & curved \\
\hline Running Speed $\left(\mathrm{m} \cdot \mathrm{s}^{-1}\right)$ & & $9.60 \pm 0.19$ & $9.63 \pm 0.14$ & $9.83 \pm 0.28$ & $* 9.49 \pm 0.27$ \\
Step frequency $(\mathrm{Hz})$ & inside & $4.59 \pm 0.27$ & $4.63 \pm 0.27$ & $4.60 \pm 0.31$ & $4.39 \pm 0.28$ \\
& outside & $4.83 \pm 0.44$ & $4.84 \pm 0.48$ & $4.32 \pm 0.20$ & $4.73 \pm 0.55$ \\
Stance time $(\mathrm{ms})$ & inside & $108.0 \pm 8.4$ & $112.0 \pm 8.8$ & $106.4 \pm 5.2$ & $116.0 \pm 6.1$ \\
& outside & $105.3 \pm 4.8$ & $104.0 \pm 3.6$ & $101.7 \pm 8.2$ & $100.0 \pm 8.0$ \\
Flight time $(\mathrm{ms})$ & inside & $110.7 \pm 10.3$ & $104.7 \pm 10.6$ & $112.3 \pm 12.4$ & $113.0 \pm 11.0$ \\
& outside & $103.3 \pm 22.3$ & $104.7 \pm 22.3$ & $130.3 \pm 7.0$ & $114.6 \pm 20.1$ \\
Step length $(\mathrm{m})$ & inside & $2.11 \pm 0.12$ & $2.09 \pm 0.14$ & $2.13 \pm 0.09$ & $2.15 \pm 0.11$ \\
& outside & $1.99 \pm 0.16$ & $2.01 \pm 0.20$ & $2.26 \pm 0.09$ & $* 2.03 \pm 0.23$
\end{tabular}

Values are expressed as the mean \pm standard deviation.

*Significant difference between straight and curved paths. 
Table 2

Differences in lower limb joint angles and angular velocities during the stance phase.

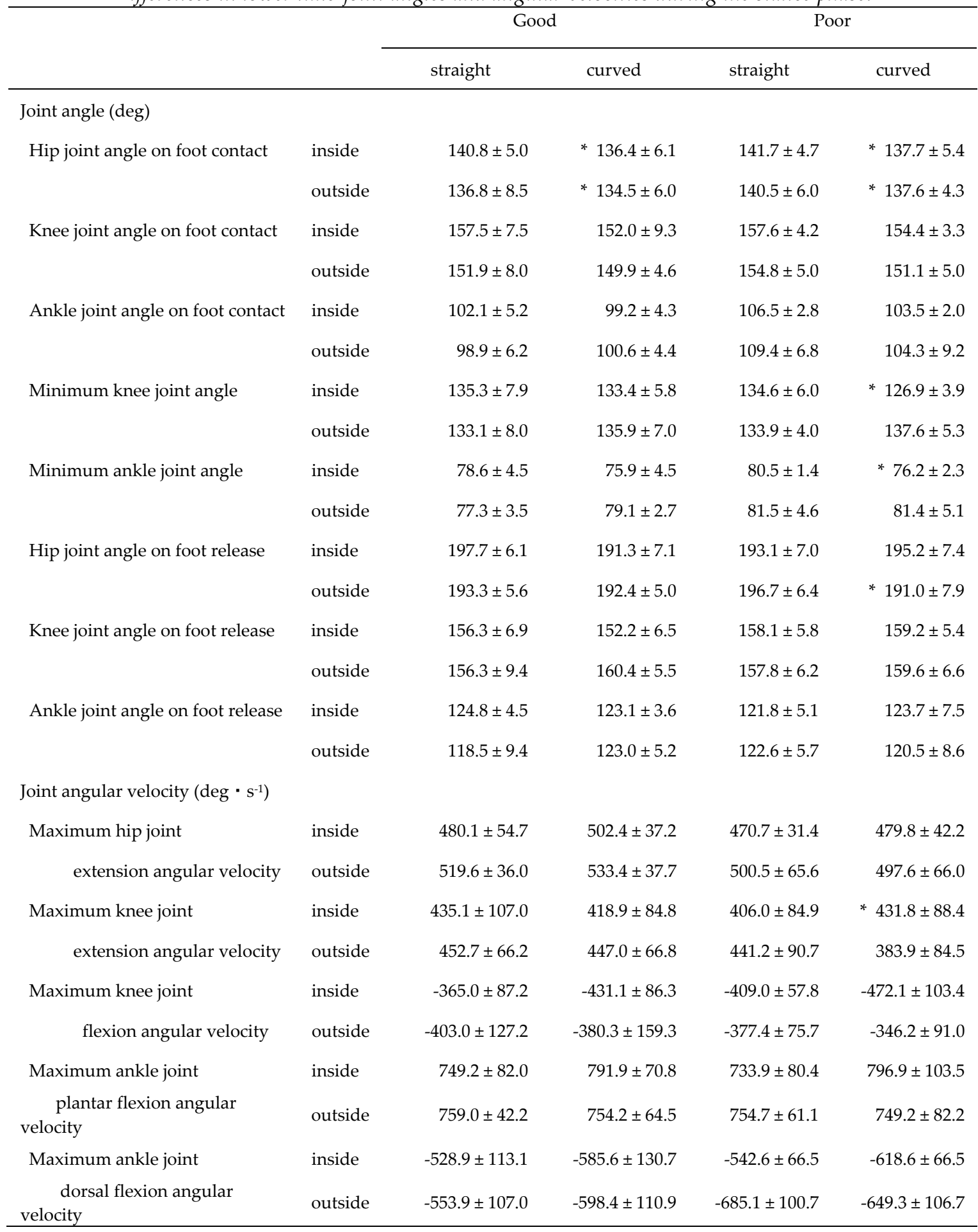

Values are expressed as the mean \pm standard deviation.

*Significant difference between straight and curved paths. 
Table 3

Differences in maximum value and impulse of ground reaction force.

\begin{tabular}{|c|c|c|c|c|c|}
\hline & \multicolumn{2}{|c|}{ Good } & \multicolumn{2}{|c|}{ Poor } \\
\hline & & straight & curved & straight & curved \\
\hline \multirow[t]{2}{*}{$\begin{array}{l}\text { Medial-lateral maximum GRF } \\
(\mathrm{N})\end{array}$} & inside & $142.5 \pm 82.5$ & $* 631.3 \pm 347.7$ & $254.1 \pm 119.6$ & * $487.5 \pm 47.9$ \\
\hline & outside & $378.3 \pm 104.7$ & *581.1 \pm 152.11 & $392.7 \pm 130.5$ & * $559.9 \pm 167.7$ \\
\hline \multirow[t]{2}{*}{$\begin{array}{l}\text { Medial-lateral minimum GRF } \\
(\mathrm{N})\end{array}$} & inside & $-437.4 \pm 101.8$ & * $-142.3 \pm 123.3$ & $-445.7 \pm 126.8$ & ${ }^{*}-166.8 \pm 77.8$ \\
\hline & outside & $-98.3 \pm 42.4$ & $*-45.5 \pm 33.0$ & $-215.4 \pm 107.6$ & * $-62.0 \pm 101.1$ \\
\hline \multirow[t]{2}{*}{ Medial-lateral impulse $(\mathrm{N} \cdot \mathrm{s})$} & inside & $2.4 \pm 3.5$ & $* 28.7 \pm 6.7$ & $1.1 \pm 5.8$ & * $27.9 \pm 11.4$ \\
\hline & outside & $-1.8 \pm 2.2$ & $* 12.2 \pm 6.8$ & $0.9 \pm 4.6$ & $* 17.0 \pm 12.7$ \\
\hline \multirow[t]{2}{*}{ Anterior peak GRF (N) } & inside & $1798.8 \pm 381.3$ & $1162.2 \pm 743.5$ & $1784.6 \pm 560.8$ & $1441.6 \pm 483.6$ \\
\hline & outside & $1691.5 \pm 380.6$ & $1363.9 \pm 509.3$ & $2169.7 \pm 424.6$ & $1638.2 \pm 382.5$ \\
\hline \multirow[t]{2}{*}{ Anterior impulse $(\mathrm{N} \cdot \mathrm{s})$} & inside & $14.2 \pm 2.4$ & $10.7 \pm 3.5$ & $15.6 \pm 2.1$ & $16.8 \pm 2.6$ \\
\hline & outside & $12.5 \pm 3.5$ & $9.5 \pm 3.1$ & $14.3 \pm 3.4$ & $13.2 \pm 4.0$ \\
\hline \multirow[t]{2}{*}{ Posterior peak GRF (N) } & inside & $-570.3 \pm 49.7$ & $-609.0 \pm 88.1$ & $-499.3 \pm 34.0$ & $-530.8 \pm 60.4$ \\
\hline & outside & $-597.4 \pm 75.4$ & $-514.3 \pm 58.3$ & $-515.3 \pm 62.0$ & * $-469.0 \pm 35.5$ \\
\hline \multirow[t]{2}{*}{ Posterior impulse $(\mathrm{N} \cdot \mathrm{s})$} & inside & $-18.3 \pm 1.5$ & $-19.5 \pm 1.5$ & $-16.2 \pm 1.2$ & $-16.7 \pm 1.5$ \\
\hline & outside & $-17.8 \pm 2.1$ & $-17.6 \pm 3.2$ & $-16.4 \pm 0.7$ & $*-14.9 \pm 0.7$ \\
\hline \multirow[t]{2}{*}{ Vertical peak GRF (N) } & inside & $2896.5 \pm 379.9$ & $2384.1 \pm 269.4$ & $2817.5 \pm 281.9$ & $2723.5 \pm 532.0$ \\
\hline & outside & $2865.3 \pm 451.7$ & $2884.2 \pm 886.0$ & $2894.5 \pm 323.1$ & $2665.3 \pm 432.0$ \\
\hline \multirow[t]{2}{*}{ Vertical impulse $(\mathrm{N} \cdot \mathrm{s})$} & inside & $144.8 \pm 13.3$ & $132.0 \pm 16.8$ & $160.5 \pm 10.1$ & $162.6 \pm 18.1$ \\
\hline & outside & $139.1 \pm 19.3$ & $119.8 \pm 31.2$ & $162.2 \pm 21.7$ & $150.6 \pm 26.1$ \\
\hline \multicolumn{6}{|c|}{$\begin{array}{l}\text { Values are expressed as the mean } \pm \text { standard deviation. } \\
\text { *Significant difference between straight and curved paths. }\end{array}$} \\
\hline
\end{tabular}
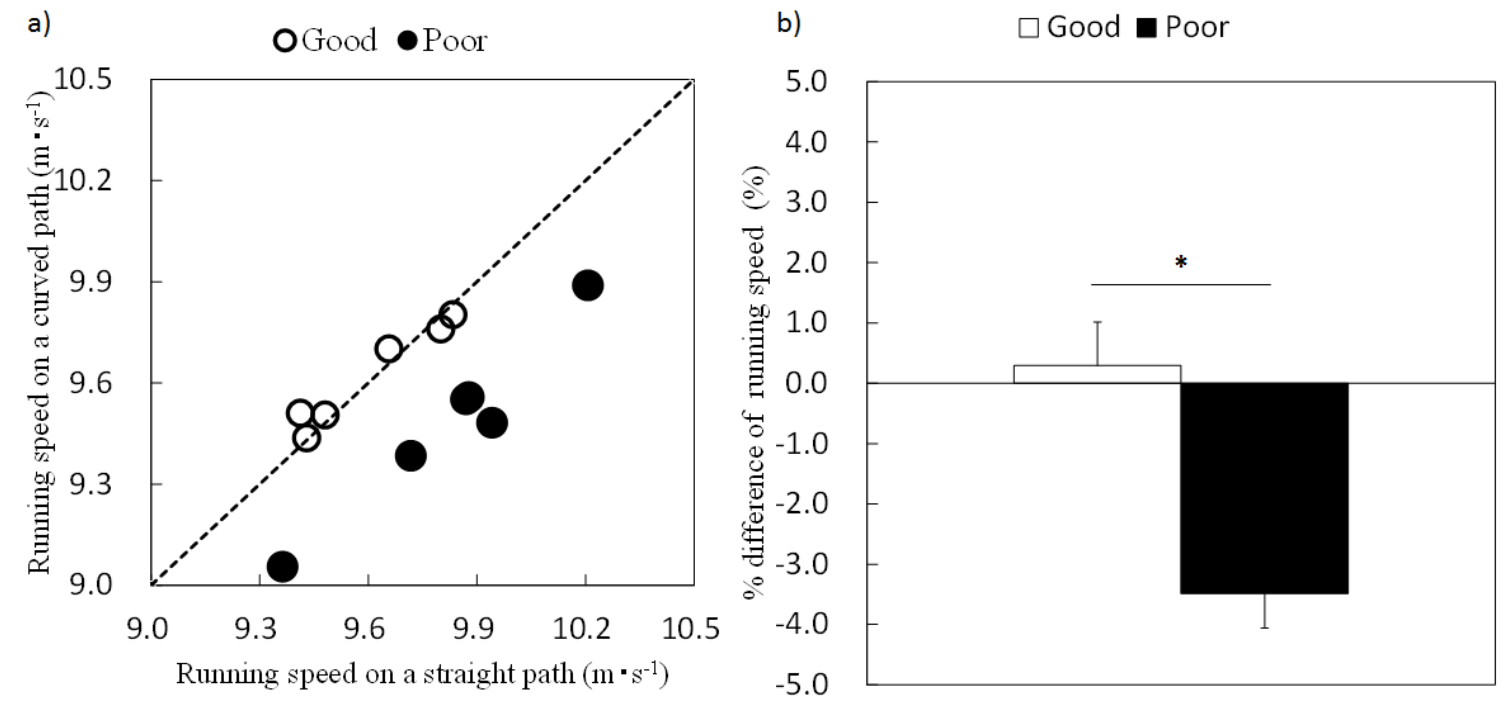

Figure 1.

a) Running speed on the curved and straight paths for each subject. The dotted line on this plot shows that running speed on curved and straight paths is in a direct proportional line.

b) Percent difference in running speed on a curved path relative to a straight path.

Percent difference in running speed in the poor curved path running group was lower

than in the good curved path running group. *Statistically significant differences. 


\section{Discussion}

Previous studies have shown that running speed on a curved path is slower than on a straight path. However, the extent of the loss in maximum running speed on a curved path differs among runners, i.e., there are good and poor curve runners. By comparing the differences in biomechanical characteristics between good and poor curve runners, this study aimed to clarify the ideal technique for running on curved paths during sprinting events.

In this study, participants were separated into good and poor curved path running groups based on running speed on a curved path relative to that on a straight path. The results suggested that running speed on a curved path was significantly slower than on a straight path in poor curve runners. However, there were no significant differences between straight and curved path running speed in good curve runners. Previous studies (Chang and Kram, 2007; Churchill et al., 2015a, 2015b; Greene, 1985; Stoner and Ben-Sira, 1979; Usherwood and Wilson, 2006) have shown that running speed on a curved path is slower than on a straight path. Additionally, this tendency becomes stronger as the curvature decreases (Chang et al., 2007). On a curved path, the medial-lateral GRF and impulse were shown to be greater than on a straight path to change direction. In this study, medial-lateral GRF and impulse on the curved path were greater than on the straight path for both groups, and there were no significant differences between groups. The medial-lateral impulse (inside leg: $28.3 \pm 8.9 \mathrm{Ns}$, outside leg: $23.8 \pm 8.0 \mathrm{Ns}$ ) during running on a curved path in this study was lower than the data (inside leg: $39.9 \pm 6.5 \mathrm{Ns}$, outside leg: $24.7 \pm 5.8 \mathrm{Ns}$ ) in a previous study (Churchill et al., 2015b). On the other hand, the vertical impulse (inside leg: $147.3 \pm 23.1 \mathrm{Ns}$, outside leg: $135.2 \pm 31.8 \mathrm{Ns}$ ) in this study was greater than the data (inside leg: $81.3 \pm$ 17.4 outside leg: $78.4 \pm 18.0 \mathrm{Ns})$ in the aforementioned study (Churchill et al., 2015b). It is suspected that there was a difference in the vector of GRFs to perform curved path running between this and the previous studies.

Although the medial-lateral GRF and impulse showed an increase during curved path running in both groups, the posterior GRF and impulse decreased only in poor curve runners. Previous studies (Brughelli et al., 2011; Morin et al., 2011, 2012) have indicated that the horizontal GRF and impulse mainly affect running speed. The results of this study suggested that running speed on a curved path was influenced only when the posterior GRF and impulse decreased. Furthermore, as shown in good curve runners, an increase in the medial-lateral GRF and impulse would not necessarily cause a decrease in the anterior-posterior GRF and impulse. In good curve runners, except for medial-lateral GRF and impulse, the analyzed variables showed no significant differences between straight and curved paths. However, in poor curve runners, some kinematic variables differed significantly between straight and curved paths. The decrease in the anterior-posterior GRF and impulse on a curved path as mentioned above could be caused by some of these differences in kinematic variables. Below, we discuss the kinematic variables that may induce the loss of running speed on a curved path.

In poor curve runners, the step length for the outside leg was significantly lower on the curved path than that on the straight path. However, there were no significant differences in the step length for the inside leg between paths. These results indicated that a decrease in step length for the outside leg caused the loss of running speed on the curved path in poor curve runners. Churchill et al. (2015a) also showed that step length on a curved path was lower than on a straight path only for the outside leg. Step length is determined by stance and flight distance (Hunter et al., 2004). Stance distance showed no significant differences between paths for both legs. Flight distance for the outside leg was significantly lower on the curved path than on the straight path. However, flight distance showed no significant differences between paths for the inside leg. Some previous studies (Brughelli et al., 2011; Hunter et al., 2004; Morin et al., 2012; Nummela et al., 2007; Weyand et al., 2000) indicated that step length and running speed were mainly determined by flight distance. The loss of running speed on curved paths in poor curve runners could be caused by the decrease in flight distance for the outside leg, which is the distance from foot release of the outside leg to foot touchdown of the inside leg. Since flight distance is decided by the impulse in stance phase, this decrease in flight distance could be 
caused by the decrease in posterior GRF and impulse. Regarding kinematic variables for the outside leg, significant differences between paths only existed in the hip joint angle at foot takeoff in poor curve runners. Therefore, the decrease of anterior-posterior GRF and impulse could affect the decrease in the hip joint extension movement during the stance phase.

For the inside leg, step length and frequency showed no significant differences between paths for both legs in this study. The minimum value of knee and ankle joint angles for the inside leg on the curved path was significantly lower than on the straight path in this study. Ryan and Harrison (2003) examined the difference in knee joint movement between inside and outside legs during the stance phase on a curved path in a 200-m sprint. Similar to this study, they reported that the minimum knee joint angle of the inside leg was larger on curved path running. They also stated that the knee joint flexion movement caused an increase of stance time for the inside leg, which could lead to the decrease in step frequency and the loss of running speed on a curved path. However, in this study, although the knee joint flexion angle of the inside leg was larger on a curved path than on a straight path, the stance time and step frequency showed no significant differences between paths. The reason for this difference could be the increase in knee joint extension velocity during the stance phase. In this study, knee joint extension velocity of the inside leg on a curved path was significantly faster than on a straight path in poor curve runners.

Several studies (Alt et al., 2015; Churchill et al., 2015a, 2015b; Hamill et al., 1987; Stoner and Ben-Sira, 1979) have focused on differences in kinematic and kinetic variables between straight and curved path running. However, the extent of the loss in maximum running speed on a curved path differs among runners, i.e., there are good and poor curve runners. In this study, we divided participants into good and poor curved path running groups and compared differences in kinematic and kinetic variables between straight and curved paths. In poor curve runners, the medial-lateral GRF and impulse on a curved path were greater than on a straight path to change direction, and the anterior-posterior GRF and impulse on a curved path were lower than on a straight path. In good curve runners, except for the medial-lateral GRF and impulse on a curved path, which were greater than on a straight path, all other analyzed variables showed no significant differences between straight and curved paths. The results of this study indicate that it is possible to run without decreasing the posterior GRF and impulse, even when the medial-lateral GRF and impulse become greater to change direction. Thus, the ideal technique for running on a curved path is to maintain the same kinematics and kinetics in the sagittal plane as on a straight path.

The number of participants in this study was limited (6 subjects in each group). Therefore, the division of participants into good and poor curve runners was clear. However, it is uncertain whether the division was appropriate. There has been no research on the relationship between running speed on the straight and curved paths. Future research with more sprinters included is required to quantify the percent difference in running speed on a curved path relative to a straight path, and evaluate the division into good and poor curve runners.

\section{Acknowledgements}

This study was conducted in Japanese Institute of Sports and Sciences. The authors received no external funding, and have no conflict of interest to disclose.

\section{References}

Ae M, Tang H, Yokoi T. Estimation of inertia properties of the body segments in Japanese athletes. Biomechanism, 1992; 11: 23-33

Alt T, Heinrich K, Funken J, Potthast W. Lower extremity kinematics of athletics curve sprinting. J Sports Sci, 2015; 33: 552-560

Brughelli M, Cronin J, Chaouachi A. Effects of running velocity on running kinetics and kinematics. J Strength Cond Res, 2011; 25: 933-939 
Chang YH, Kram R. Limitations to maximum running speed on flat curves. J Exp Biol, 2007; 210: 971-982

Churchill SM, Salo AI, Trewartha G. The effect of the bend on technique and performance during maximal effort sprinting. Sports Biomech, 2015; 14: 106-121

Churchill SM, Trewartha G, Bezodis IN, Salo AI. Force production during maximal effort bend sprinting: Theory vs reality. Scand J Med Sci Sports, 2015; 26: 1171-1179

Cohen J. A power primer. Psychol Bull, 1992; 112: 155-159

Davis RB, Ônpuu S, Tyburski D, Gage JR. A gait analysis data collection and reduction technique. Hum Mov Sci, 1991; 10: 575-587

Greene PR. Running on flat turns; experiments, theory, and applications. J Biomech Eng, 1985; 107: 96-103

Hamill J, Murphy M, Sussman D. The Effects of Track Turns on Lower Extremity Function. Int J Sport Biomech, 1987; 3: 276-286

Hunter JP, Marshall RN, McNair PJ. Interaction of step length and step rate during sprint running. Med Sci Sports Exerc, 2004; 36: 261-271

Kadaba MP, Ramakrishnan HK, Wootten ME. Measurement of lower extremity kinematics during level waking. J Orthop Res, 1990; 8: 383-392

Luo G, Stefanyshyn D. Ankle moment generation and maximum-effort curved sprinting performance. J Biomech, 2011; 45: 2763-2768

Mackala K. Optimisation of performance through kinematic analysis of the different phases of the 100 metres. New Studies in Athletics, 2007; 22: 7-16

Mero A, and Komi PV. Effects of Supramaximal Velocity on Biomechanical Variables in Sprinting. Int J Sport Biomech, 1985; 1: 240-252

Morin JB, Bourdin M, Edouard P, Peyrot N, Samozino P, Lacour JR. Mechanical determinants of 100-m sprint running performance. Eur J Appl Physiol, 2012; 112: 3921-3930

Morin JB, Edouard P, Samozino P. Technical ability of force application as a determinant factor of sprint performance. Med Sci Sports Exerc, 2011; 43: 1680-1688

Nummela A, Keranen T, Mikkelsson LO. Factors related to top running speed and economy. Int J Sports Med, 2007; 28: 655-661

Quinn MD. The effect of track geometry on 200- and 400-m sprint running performance. J Sports Sci, 2009; 27: 19-25

Ryan GJ, Harrison AJ. Technical adaptations of competitive sprinters induced by bend running. New Studies in Athletics, 2003; 18: 57-67

Smith N, Dyson R, Hale T, Janaway L. Contributions of the inside and outside leg to maintenance of curvilinear motion on a natural turf surface. Gait Posture, 2006; 24: 453-458

Stoner LJ, Ben-Sira D. Science in Athletics. Del Mar: Academic publisher, 167-173; 1979

Usherwood JR, Wilson AM. Accounting for elite indoor $200 \mathrm{~m}$ sprint results. Biolo Lett, 2006; 2: 47-50

Weyand PG, Sternlight DB, Bellizzi MJ, Wright $S$. Faster top running speeds are achieved with greater ground forces not more rapid leg movements. J App Physiol, 2000; 89: 1991-1999

\section{Corresponding author:}

\section{Hayato Ohnuma, Ph.D}

Japan Institute of Sports Science

Department of Sports Science

3-15-1, Nishigaoka, Kita-ku, Tokyo, Japan, 115-0056

Phone number: 81-3-5963-0200

Fax number: 81-3-5963-0244

E-mail: hayato.onuma@jpnsport.go.jp 